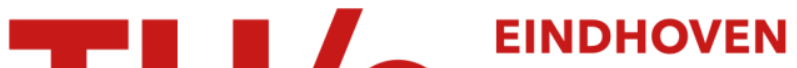 UNIVERSITY OF TECHNOLOGY
}

\section{Radio-over-MMF techniques - Part II: microwave to millimeter- wave systems}

Citation for published version (APA):

Koonen, A. M. J., \& Garcia Larrode, M. (2008). Radio-over-MMF techniques - Part II: microwave to millimeterwave systems. Journal of Lightwave Technology, 26(15), 2396-2408. https://doi.org/10.1109/JLT.2008.927182

DOI:

10.1109/JLT.2008.927182

Document status and date:

Published: 01/01/2008

Document Version:

Publisher's PDF, also known as Version of Record (includes final page, issue and volume numbers)

Please check the document version of this publication:

- A submitted manuscript is the version of the article upon submission and before peer-review. There can be important differences between the submitted version and the official published version of record. People interested in the research are advised to contact the author for the final version of the publication, or visit the $\mathrm{DOI}$ to the publisher's website.

- The final author version and the galley proof are versions of the publication after peer review.

- The final published version features the final layout of the paper including the volume, issue and page numbers.

Link to publication

\section{General rights}

Copyright and moral rights for the publications made accessible in the public portal are retained by the authors and/or other copyright owners and it is a condition of accessing publications that users recognise and abide by the legal requirements associated with these rights.

- Users may download and print one copy of any publication from the public portal for the purpose of private study or research.

- You may not further distribute the material or use it for any profit-making activity or commercial gain

- You may freely distribute the URL identifying the publication in the public portal.

If the publication is distributed under the terms of Article $25 \mathrm{fa}$ of the Dutch Copyright Act, indicated by the "Taverne" license above, please follow below link for the End User Agreement:

www.tue.nl/taverne

Take down policy

If you believe that this document breaches copyright please contact us at:

openaccess@tue.nl

providing details and we will investigate your claim. 


\title{
Radio-Over-MMF Techniques-Part II: Microwave to Millimeter-Wave Systems
}

\author{
A. M. J. Koonen, Fellow, IEEE, and M. García Larrodé, Student Member, IEEE
}

(Invited Paper)

\begin{abstract}
Microwave to mm-wave radio carriers are commonly employed for creating high-capacity picocell wireless networks. Advanced radio-over-fiber (RoF) techniques can efficiently generate and transport such carriers, and deliver them to simplified antenna stations. As in in-building networks multimode fiber is predominantly used, adequate radio-over-multimode fiber (RoMMF) techniques are required to overcome the modal dispersion in multimode fiber links. The optical frequency multiplying technique is introduced; it is relatively simple to implement, yet it is shown to be robust against the modal dispersion, and it is able to generate very pure microwave carriers while requiring only moderate speed electronics. Thus, it can convey high data rates in comprehensive modulation formats on multiple-GHz carriers in MMF networks. It offers simultaneous operation at multiple radio standards, and capabilities for dynamic adaptation of the radio link parameters such as carrier frequency, transmit power, and other antenna site functions by means of an embedded control channel. Moreover, in combination with optical routing it enables dynamically adjustable network configurations for flexible wireless service delivery.
\end{abstract}

Index Terms-Broadband wireless access, microwave radio communication, multimode fiber (MMF), optical fiber communication, radio-over-fiber $(\mathrm{RoF})$.

\section{INTRODUCTION}

A FTER the installation of fiber as deep as possible into the access network, i.e., up to the doorstep of the home in an FTTH topology, the next challenge is to extend its broadband capacity into the residential home [1]. More in general, this also concerns other buildings such as hospitals, office buildings, conference centers, airport departure lounges, etc. In those networks, the demand for broadband services for wirebound as well as wireless devices is rapidly growing, fueled by broadband internet, fast peer-to-peer file transfer, high definition video, on-line multiparty gaming, etc. Gbit/s wirebound capacity is

Manuscript received February 4, 2008; revised May 16, 2008. Current version published October 10, 2008. This work was supported in part by the European Commission in the projects FP6 e-Photon/ONe, FP7 ALPHA, and FP7 BONE and by the Dutch Ministry of Economic Affairs in the IOP Generieke Communicatie projects "Broadband wireless in-house networks employing radio over multimode fiber" and "Future Home Networks".

The authors are with the COBRA Institute, Electro-Optical Communication Systems Group, Department of Electrical Engineering, Eindhoven University of Technology, NL 5600MB, Eindhoven, The Netherlands (e-mail: a.m.j.koonen@tue.nl; m.garcia.larrode@tue.nl).

Color versions of one or more of the figures in this paper are available online at http://ieeexplore.ieee.org.

Digital Object Identifier 10.1109/JLT.2008.927182 needed to feed fixed terminals, such as desktop PC-s, (HD)TV sets, scanners, printers, local storage servers, etc. In addition, a large wireless capacity is needed. High carrier frequencies of $10 \mathrm{GHz}$ and above are required, and many radio picocells. For example, the IEEE 802.16-2004 standard specifies frequencies between 10 and $66 \mathrm{GHz}$ for Fixed Wireless Access, and can deliver up to $100 \mathrm{Mbit} / \mathrm{s}$. The IEEE $802.15 .3 \mathrm{c}$ standard can provide up to $3 \mathrm{Gbit} / \mathrm{s}$ over short ranges in Wireless Personal Area Networks, with carrier frequencies between 57 and $64 \mathrm{GHz}$.

Typically, the wired as well as wireless services are being delivered by a number of dedicated networks (CAT5 cables, coaxial cables, copper twisted pair cables, WLAN, DECT, etc.). A single in-building infrastructure which can host all these services together would considerably reduce installation and maintenance costs, and ease the introduction and upgrading of (new) services. Optical fiber is the ideal medium for such a converged network, due to its signal transparency and wide bandwidth. Single-mode fiber (SMF) requires delicate handling and skilled personnel for installation, and is, hence, relatively expensive to install. For in-building networks, multimode fiber (MMF) is predominantly used worldwide [2]. MMF is easier to install than SMF, as its core size (typically 50 or $62.5 \mu \mathrm{m}$ ) is significantly larger, which makes splicing and connectorizing much simpler and more efficient. It is also easier to couple to transceiver modules, which brings considerable cost savings. In particular, polymer optical fiber (POF) is easy to install, thanks to its ductility when pulling through ducts. Connectorizing and splicing of POF can be readily realized, by cutting the fiber with, e.g., a razor blade and directly cramping a metal connector ferrule on it without a delicate UV glue curing process. Very large core POF, such as 1-mm-core diameter step-index PMMA POF, is even becoming suited for do-it-yourself installation, which is expected to give the real breakthrough for low-cost in-building optical networks. PMMA POF has significantly higher losses than silica fiber. It is only transparent in the visible light wavelength region, with minimum losses of $0.1 \mathrm{~dB} / \mathrm{m}$ at $560 \mathrm{~nm}$. However, as link lengths are short in in-building networks, typically less than $200 \mathrm{~m}$, high fiber losses do not overshadow the benefits of easy installation. The use of visible light also eases inspection of the system's operational status during its installation and operation.

The converged in-building network should host wired as well as wireless services, and should operate with a variety of standards. Fig. 1 illustrates how such a network may deploy a common (polymer) optical fiber backbone, to which both wired 


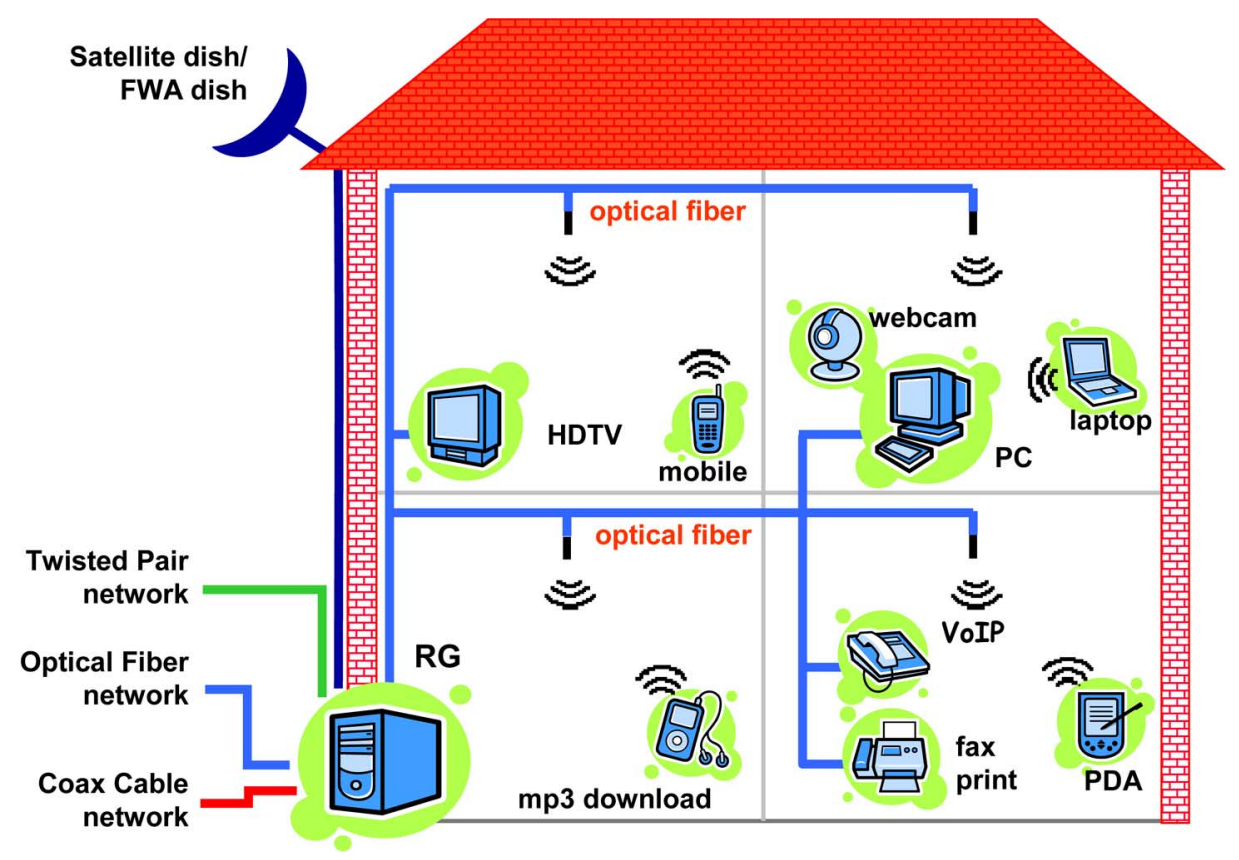

Fig. 1. In-building network using optical fiber (RG: residential gateway).

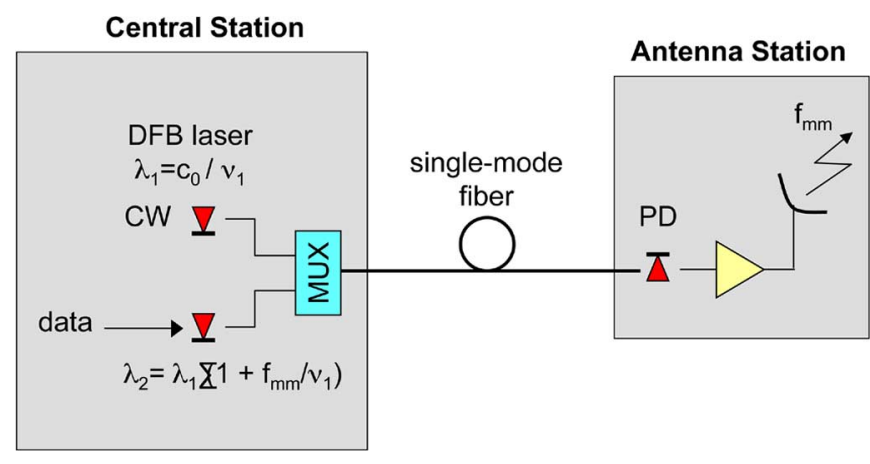

Fig. 2. Optical heterodyning with two sources.

and wireless terminals are hooked up. In order to achieve a high capacity per user and thus decrease the sharing factor per wireless cell, a reduction of the cell size is needed. Picocells can be created by using high-frequency radio carriers in the $\mathrm{mm}$-wave range, as these have high propagation losses and are not able to penetrate walls; thus, they are easily confined to a single room minimizing interfering with cells in adjacent rooms.

The interfacing of the in-building network with the access network can take place via the residential gateway (RG), which contains media converters and possibly additional intelligence for signal conversion, local data storage, security and authorization functions, etc.

Due to the modal dispersion, in particular in step-index fibers, the nominal bandwidth of large-core POF is quite limited. Advanced signal processing and multiplexing techniques are therefore required to accommodate multiple services in an in-building network. And as aforementioned, although its attenuation per unit length is far higher than that of silica fiber, it does not represent a major concern in in-building networks, where short links are deployed.

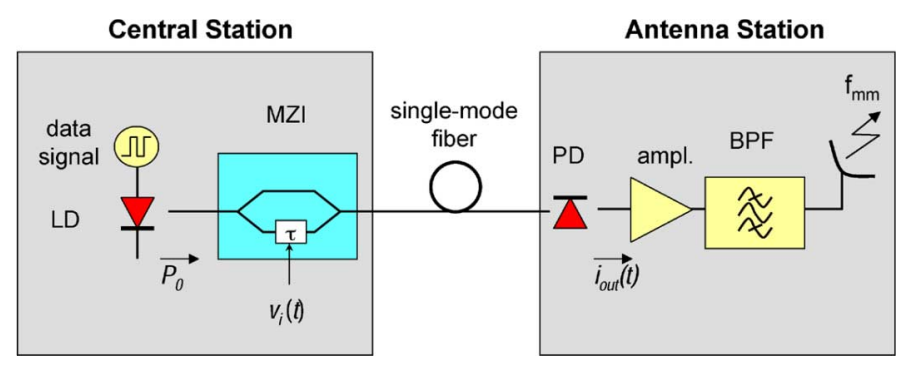

Fig. 3. Optical heterodyning with a single source.

Many research efforts have been dedicated to develop low-cost radio-over-multimode-fiber (RoMMF) for distributed antenna systems with short multimode fiber distances [3]-[6]. Most of these techniques have been successfully demonstrated in point-to-point links for wireless services below $2.5 \mathrm{GHz}$, making use of new high-bandwidth multimode fibers like in [4], or taking advantage of the passband transmission regions of a multimode fiber link for wireless services beyond $5 \mathrm{GHz}$, like in [6]. Here the distributed antenna system (DAS) is commonly implemented as a bunch of RoMMF point-to-point links. An extensive review of the advances in this field is given in the Part I companion paper to this paper [7]. However, for carrying microwave and $\mathrm{mm}$-wave wireless services in a point-to-multipoint multimode fiber network, RoMMF techniques which are robust against multimodal dispersion are required. After reviewing nonexhaustively some techniques for optically generating microwave and $\mathrm{mm}$-wave signals, this paper will focus on the optical frequency multiplying technique, which is shown to be particularly suited for multimode fiber.

\section{RAdio-Over-Fiber (RoF) Microwave Techniques}

The transmission of multi-GHz radio signals over fiber by means of direct RF intensity modulation of the optical source 


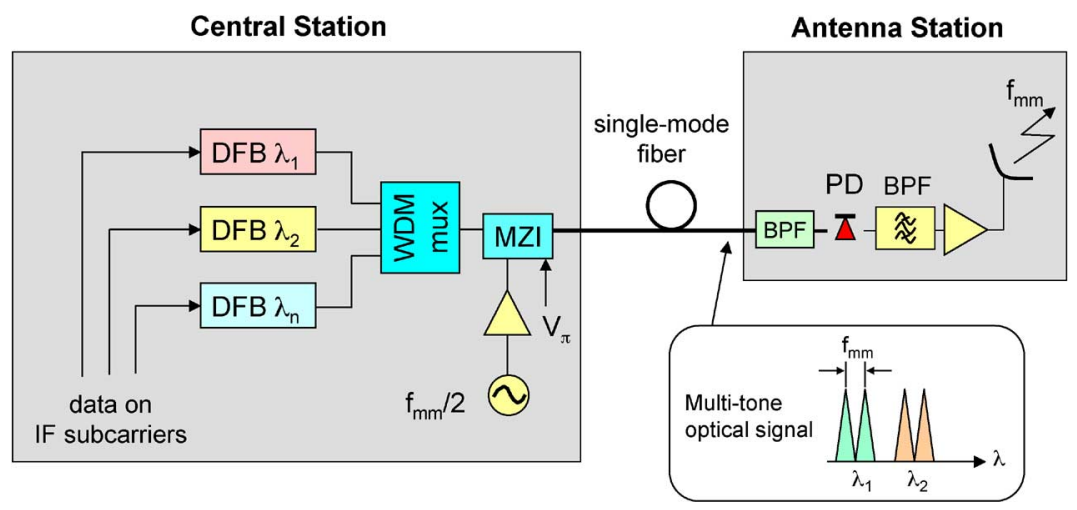

Fig. 4. Generating multiple microwave signals by optical heterodyning.

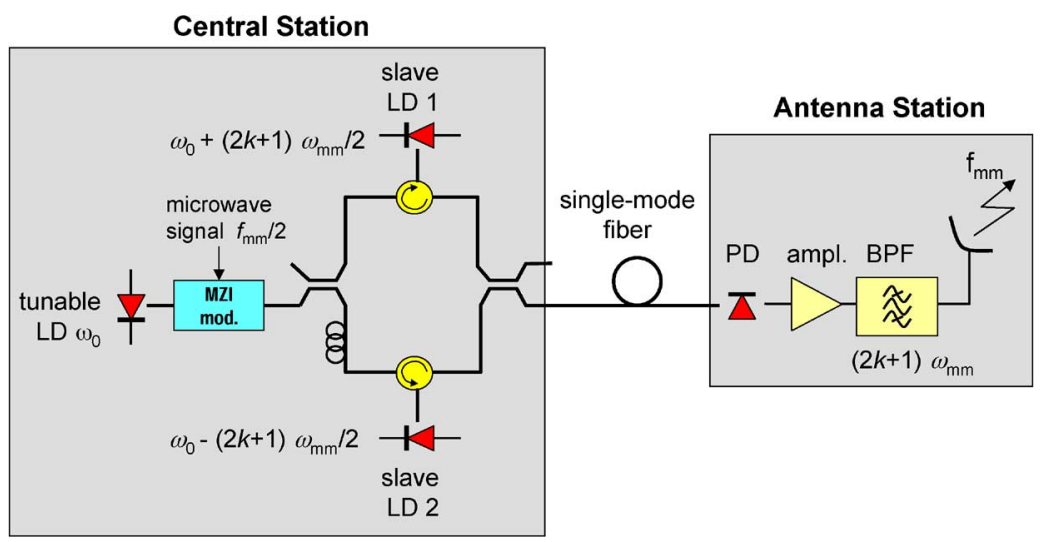

Fig. 5. Microwave signal generation by optical injection locking and heterodyning.

requires that the optical transmitter and receiver can operate adequately at very high frequencies. Also, careful fiber dispersion techniques may be needed. Depending on the signal modulation format, the modulation and demodulation processes must meet high linearity requirements. These challenges can be met efficiently by using optical techniques for generating the microwave carrier and for modulating it. Many approaches have been reported in literature, which may be classified into methods deploying the heterodyning process of optical carriers, and into methods deploying the generation of high-frequency harmonics by optical nonlinear processes such as FM-to-IM conversion.

In this section, some approaches are reviewed which are based on optical heterodyning. The heterodyning process requires polarization alignment of the associated optical carriers, which may be disrupted in multimode fiber links. Hence, in the next section an alternative approach is discussed which is based on FM-to-IM conversion. This approach, termed optical frequency multiplication (OFM), does not require such polarization alignment, and is shown to be robust against modal dispersion in multimode fiber.

An alternative to the transport of a modulated microwave radio signal through the fiber is to remotely generate the modulated microwave signal by optical heterodyning. Fig. 2 shows the principle. Two laser diodes with narrow spectral linewidth are used, of which the central optical frequency spacing equals the microwave frequency, and one of them is intensity-modulated with the data whereas the other laser is emitting continuous-wave light. The light of both lasers is combined and transmitted over the fiber, where at the receiver by means of optical heterodyning in the photodiode the microwave radio signal emerges, amplitude-modulated with the data signal. After a simple amplifier, the modulated microwave is radiated by an antenna. In this way, the antenna station (AS) can be very simple, and thus low-cost, cheap to install, and the power consumption is reduced. All the complex signal processing can be consolidated at the central station (CS). The spectral linewidth of the microwave signal equals the sum of the linewidths of the laser diodes. As the microwave carrier must be quite pure in order to meet the requirements of the commonly used multilevel multiphase signal formats [such as multilevel Quadrature Amplitude Modulation (QAM)], the linewidths of the laser diodes should be very small; this may be achieved by injection locking, as discussed at the end of this section. Also the difference in optical emission frequency of the lasers must be carefully stabilized, in order to get a stable microwave frequency.

An approach which uses only a single laser diode is shown in Fig. 3. The subsequent Mach Zehnder Interferometer modulator (MZI) is biased at the inflexion point $\left(\mathrm{V}_{\pi}\right)$ of its modulation characteristic, and is driven by a sinusoidal signal at half the desired microwave frequency [8]. Thus, at the output of the MZI a two-tone optical signal emerges with a tone spacing which is equal to the microwave frequency, and with suppressed optical carrier. When intensity-modulating the laser with the data signal, self-heterodyning in the photodiode at the receiver yields the modulated microwave signal. 
Central Station

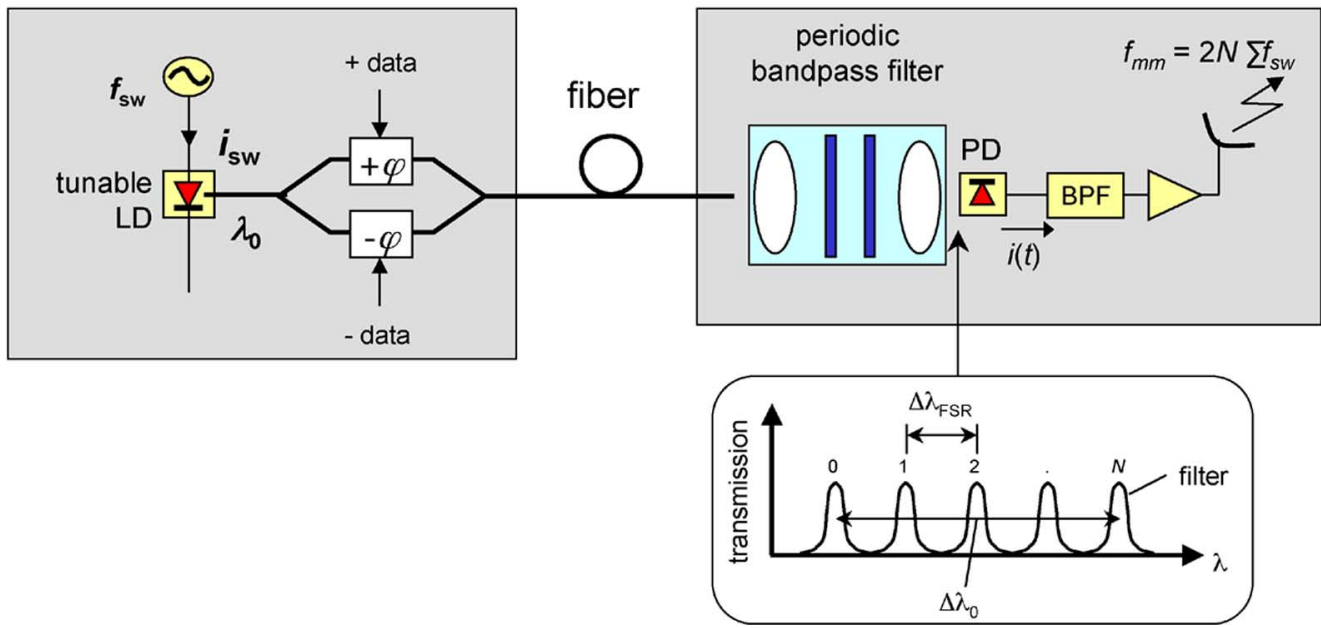

Fig. 6. Remote generation of microwave signals using OFM.

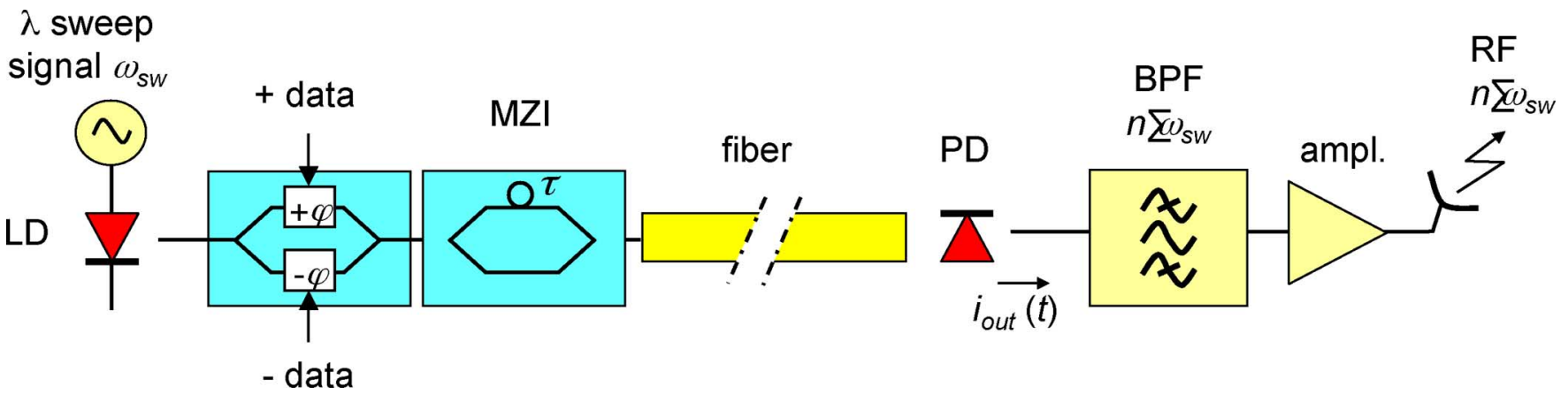

Fig. 7. Point-to-point OFM system using a MZI periodic filter.

When assuming that the laser diode has a central optical frequency $\omega_{0}$ and an average output power $P_{0}$, and that the MZI's delay $\tau$ is modulated by the external signal $v_{i}(t)$ sinusoidally with an amplitude $\tau_{\mathrm{sw}}$ and a frequency $\omega_{\mathrm{sw}}$, neglecting the fiber losses the output current $i_{\text {out }}(t)$ of the photodiode having a responsivity $R_{d}$ is [9]

$$
\begin{aligned}
i_{\text {out }}(t)=R_{d} P_{0}\{1- & J_{0}\left(2 \omega_{0} \tau_{\mathrm{sw}}\right) \\
& \left.\quad-2 \sum_{n=1}^{\infty} J_{2 n}\left(2 \omega_{0} \tau_{\mathrm{sw}}\right) \cos 2 n \omega_{\mathrm{sw}} t\right\}
\end{aligned}
$$

The signal $i_{\text {out }}(t)$ thus contains even harmonics of the sweep frequency $\omega_{\text {sw }}$, and the $2 n$-th harmonic has an amplitude $2 R_{d} P_{0} J_{2 n}\left(2 \omega_{0} \tau_{\mathrm{sw}}\right)$, where $J_{n}(x)$ denotes the Bessel function of the first kind of order $n$. It can also be shown that the requirements on the laser diode's linewidth are relaxed, as the phase noise of the optical source is effectively suppressed provided that the optical source linewidth is much smaller than the MZI's free spectral range. Hence, this method can produce pure microwave carriers by generating harmonics of the relatively low modulating sweep frequency. The system is also quite tolerant to dispersion in the single mode fiber link, as the heterodyned sidebands are located relatively close to each other in the optical spectrum.
Based on the approach of Fig. 3, the transmitter site may use multiple laser diodes for transmitting multiple radio channels, as shown in Fig. 4, [8]. The data signals are modulated on IF subcarriers, which each are intensity-modulated on a DFB laser operating at a different wavelength. In the MZI, the wavelength-multiplexed channels are each converted in a two-tone suppressed-carrier optical signal. At the antenna station, by means of a (tunable) optical bandpass filter one of the wavelength channels can be selected, and by self-heterodyning converted in the microwave radio signal.

One can obtain a very clean microwave signal by injection locking two lasers on specific sidebands selected out of the many ones generated by the MZI by means of the method shown in Fig. 3, [10]. As illustrated in Fig. 5, each slave laser diode is phase-locked to a specific sideband by tuning (e.g., by temperature) the laser close to this tone. The two tones at which the slave laser diodes are lasing are separated by the desired microwave frequency. The resulting microwave carrier after heterodyning in the photodiode at the receiver is of high purity and high power.

\section{Optical Frequency Multiplying (OFM) Technique}

As discussed, the techniques considered in Section II are not suitable for MMF-based networks, as they rely on heterodyning. Hence, for these networks another technique has been proposed 


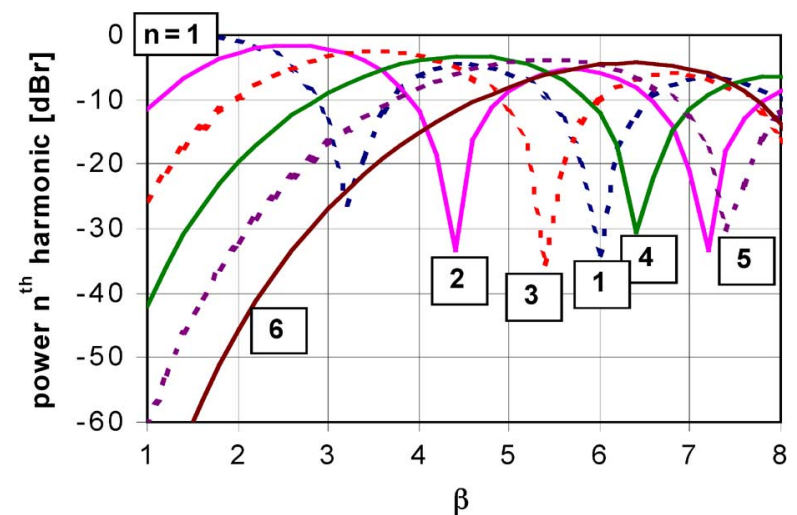

Fig. 8. Impact of the FM modulation index $\beta$ on the power of the microwave frequency components ( $n$th harmonics of the sweep frequency) generated by OFM using an MZI periodic filter.

\section{Central Station}

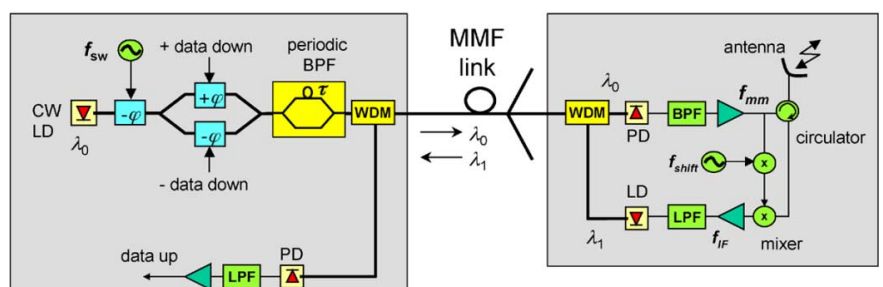

Fig. 9. Bidirectional transmission.

in [11], which has been named OFM. The basic concept is shown in Fig. 6. In the CS, the wavelength of a tunable optical source is periodically swept over a range $\Delta \lambda_{0}$. At the antenna station (AS), a periodic optical bandpass filter is deployed (such as a Fabry-Perot filter), of which the bandpass transmission peaks are spaced by the wavelength free spectral range $\Delta \lambda_{\mathrm{FSR}}$. Linearly sweeping the source wavelength back and forth with a sweep frequency $f_{\text {sw }}$ across $N$ bandpass transmission peaks, yields light intensity bursts on the photodiode (PD) each time when a peak is traversed, and, thus, a microwave signal is generated with the fundamental frequency $2 N \cdot f_{\text {sw }}$ and also higher harmonics [12]. The electrical bandpass filter (BPF) selects the particular harmonic for radiation by the antenna. The data signal is chirp-free intensity-modulated on the frequency-swept optical carrier by means of, e.g., a differentially driven Mach-Zehnder modulator. This intensity-modulated signal is not affected by the OFM process, and appears as the envelope of the swept optical field before photodetection, and, hence, it is transparently upconverted along with the harmonic microwave after photodetection.

When multimode fiber is used, modal dispersion occurs due to the different propagation times of the many individual guided modes. As the fiber is assumed to behave linearly with respect to transporting the electrical fields, one can also put the periodic filter at the CS instead of at the AS, so first do the FM-to-IM conversion in the filter and then the transmission by the fiber, without affecting the OFM process. This also facilitates the alignment of the filter with the wavelength sweep range of the laser diode.

Basically, there are various options to realise the optical periodic bandpass filter, such as a fiber Fabry-Perot filter, a fiber
Bragg grating (FBG) filter, or a MZI filter. When the fiber network is passively split, the filter can be shared by a number of AS-s, and, thus, the system's costs are lowered. The periodic filter may be equipped with single-mode fiber pigtails, and may even be integrated within the tunable source module. Furthermore, the periodic filter can be locally controlled and tuned to the source's sweep behavior, which helps optimizing system performance and eases system operation and upgrades.

\section{A. OFM System Analysis}

Deploying an MZI periodic filter positioned at the CS, the point-to-point OFM system can be modeled as shown in Fig. 7.

Assuming that the optical frequency of the laser diode is harmonically swept at a radian sweep frequency $\omega_{\text {sw }}$ over an optical radian frequency range $2 \beta \cdot \omega_{\text {sw }}$ around a central optical frequency $\omega_{0}$, that the laser light has a phase noise $\varphi(t)$, and that $\tau$ is the time delay difference between the two MZI arms (so its bandpass maxima are spaced at its free spectral range $\left.\Delta \Omega_{\mathrm{FSR}}=2 \pi / \tau\right)$, it can be derived that neglecting fiber dispersion the output current signal $i_{\text {out }}(t)$ of the photodiode is [9]

$$
\begin{aligned}
i_{\mathrm{out}}(t)=R_{d} P_{0} & \cdot\left\{1+\cos \left[2 \beta \cdot \sin \left(\frac{1}{2} \omega_{\mathrm{sw}} \tau\right)\right.\right. \\
\cdot & \left.\left.\cos \left(\omega_{\mathrm{sw}}\left(t-\frac{1}{2} \tau\right)\right)+\omega_{0} \tau+\dot{\varphi} \tau\right]\right\} .
\end{aligned}
$$

The signal $i_{\text {out }}(t)$ thus contains even harmonic frequency components $2 n \cdot \omega_{\mathrm{sw}}$ with amplitude $2 R_{d} P_{0} \cos \left(\omega_{0} \tau+\right.$ $\dot{\varphi} \tau) \cdot J_{2 n}\left(2 \beta \cdot \sin \left((1 / 2) \omega_{\mathrm{sw}} \tau\right)\right)$, and odd harmonic frequency components $(2 n-1) \cdot \omega_{\mathrm{sw}}$ with amplitude $2 R_{d} P_{0} \sin \left(\omega_{0} \tau+\dot{\varphi} \tau\right) \cdot J_{2 n-1}\left(2 \beta \cdot \sin \left((1 / 2) \omega_{\mathrm{sw}} \tau\right)\right)$. The harmonics are spaced at $\omega_{\mathrm{sw}}$, and, hence, using double-sideband modulation each harmonic can carry data signals with a maximum bandwidth of $1 / 2 \omega_{\mathrm{sw}}$.

The relative power of the harmonic components as a function of the optical FM modulation index $\beta$ is shown in Fig. 8, assuming a sweep frequency $f_{\mathrm{sw}}=\omega_{\mathrm{sw}} / 2 \pi=2 \mathrm{GHz}$, and an MZI free spectral range $\Delta \nu_{\mathrm{FSR}}=\Delta \Omega_{\mathrm{FSR}} / 2 \pi=10 \mathrm{GHz}$. Obviously, a higher FM index $\beta$ is needed to achieve the maximum signal power at higher harmonic frequencies. For example, $\beta \approx$ 6.3 is needed to achieve the maximum power generated in the sixth harmonic of the sweep frequency (i.e., at $12 \mathrm{GHz}$ ).

The laser frequency noise (represented by $\dot{\varphi}=d \varphi / d t$ ) has negligible impact if $\dot{\varphi} \tau \ll \pi / 2$. So if the laser linewidth is much smaller than a quarter of the MZI's Free Spectral Range $\Delta \omega_{\mathrm{FSR}}=2 \pi / \tau$, the OFM process effectively suppresses the impact of the laser phase noise. Thus, by using the OFM method microwave signals with very low phase noise can be generated without requiring a narrow laser linewidth. Experiments have shown extremely narrow microwave spectral linewidths, below the measurement resolution; see Section V. These pure microwave signals allow comprehensive signal constellations, and therewith high-capacity wireless transmission.

\section{B. Impact of Dispersion in Multimode Fiber Systems}

The impulse response of a multimode fiber, neglecting mode coupling and chromatic dispersion (since the fiber's dispersion is strongly dominated by its modal dispersion), and taking into 


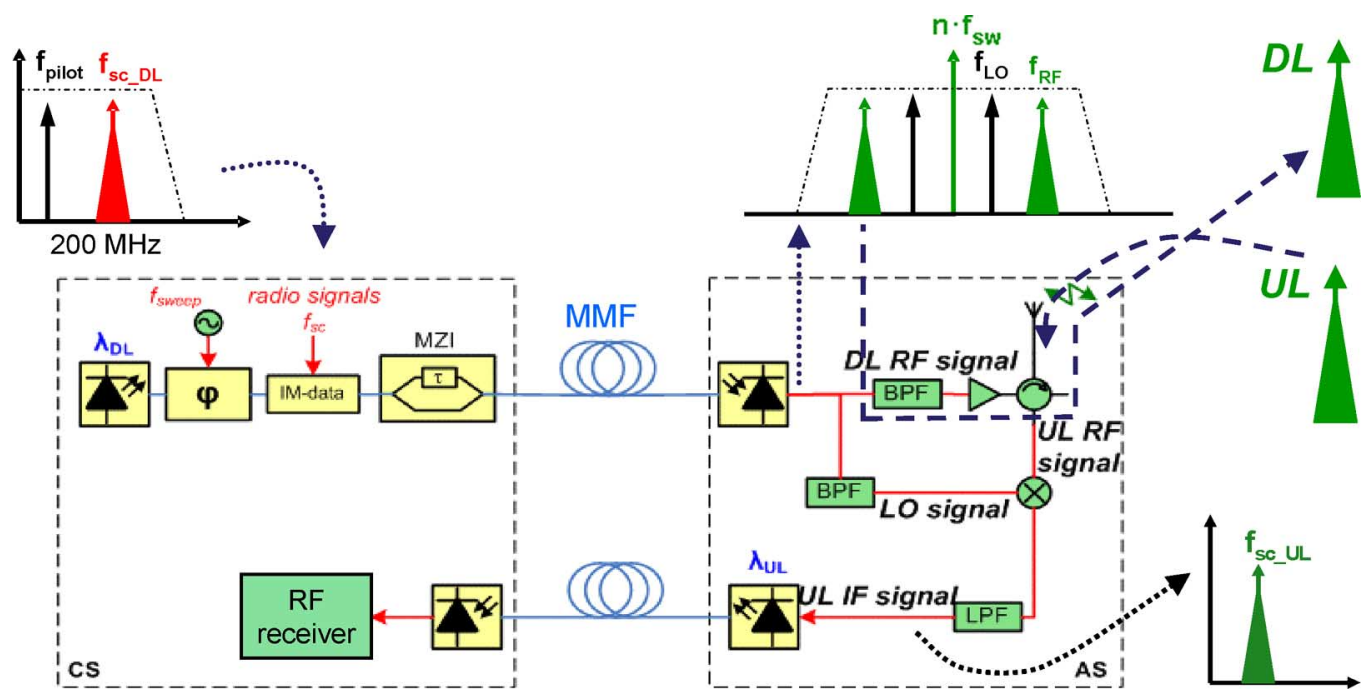

Fig. 10. Bidirectional OFM transmission with remote LO delivery (DL: downlink, UL: uplink).

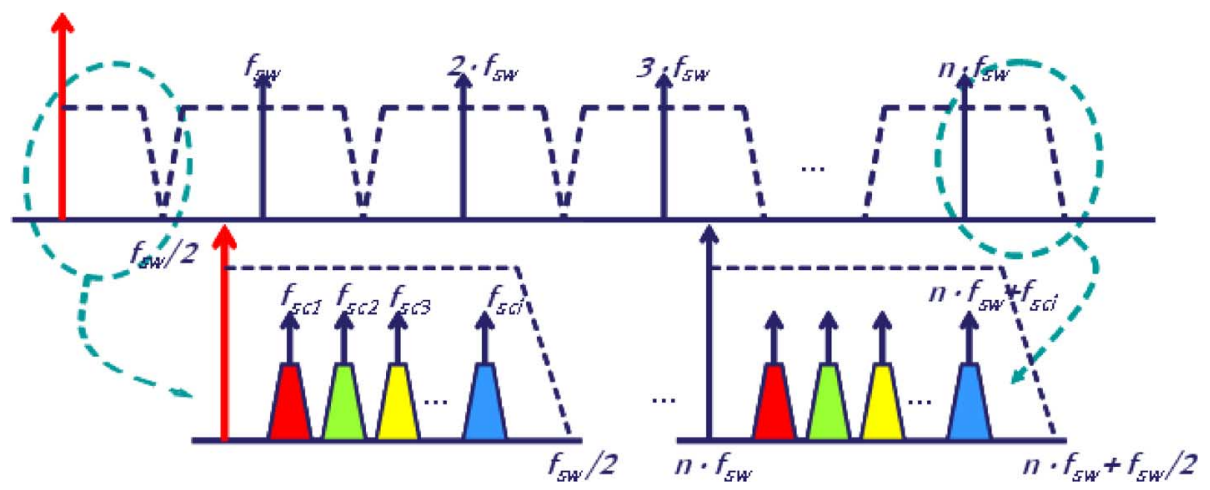

Fig. 11. RF bandwidth capacity in OFM.

account the delay times $\tau_{i}$ of the $M$ individual guided modes $(i=1 \cdots M)$ and a mode group power distribution $c_{i}$ of the input electrical field to the $i$ th mode, can be denoted as

$$
h_{\mathrm{MMF}}(t)=\sum_{i=1}^{\mathrm{M}} \mathrm{c}_{i} \delta\left(t-\tau_{i}\right)
$$

by which the relation between the electrical fields at the output and the input of the fiber is given by

$$
E_{\text {out }}(t)=E_{\text {in }}(t) * h_{\mathrm{MMF}}(t) .
$$

Using the orthogonality of the modes and introducing an intensity-modulation fiber impulse response $h_{\mathrm{IM}}(t)$, the relation between the light intensity signals at the output and the input of the fiber is then

$$
P_{\text {out }}(t)=P_{\text {in }}(t) * h_{\mathrm{IM}}(t)=P_{\text {in }}(t) * \sum_{i=1}^{\mathrm{M}} \mathrm{c}_{i}^{2} \delta\left(t-\tau_{i}\right)
$$

with which an intensity modulation transfer function of the fiber can be defined as

$$
H_{\mathrm{IM}}(\omega)=\mathcal{F}\left(h_{\mathrm{IM}}(t)\right)=\sum_{i=1}^{M} c_{i}^{2} e^{-j \omega \tau_{i}} .
$$

Using this modal dispersion model, it can be shown that the output current $i_{\text {out }}(t)$ of the photodiode is given by (7), shown at the bottom of the page [9], where $P_{0}$ denotes the average received optical power and $R_{d}$ the responsivity of the photodiode.

$$
\begin{aligned}
i_{\text {out }}(t)= & \frac{1}{2} R_{d} P_{0} \cdot \sum_{i=1}^{M} c_{i}^{2} \cdot\left\{1+\cos \omega_{0} \tau \cdot J_{0}\left(2 \beta \cdot \sin \left(\frac{\omega_{m} \tau}{2}\right)\right)\right\} \\
& +R_{d} P_{0} \cdot \sum_{n=1}^{\infty}(-1)^{n}\left\{\sum_{i=1}^{M} c_{i}^{2} \cdot\left\{\begin{array}{c}
\cos \omega_{0} \tau \cdot J_{2 n}\left(2 \beta \cdot \sin \left(\frac{\omega_{m} \tau}{2}\right)\right) \cdot \cos \left[2 n \cdot\left(\omega_{m}\left(t-\tau_{i}\right)-\frac{\omega_{m} \tau}{2}\right)\right] \\
+\sin \omega_{0} \tau \cdot J_{2 n-1}\left(2 \beta \cdot \sin \left(\frac{\omega_{m} \tau}{2}\right)\right) \cdot \cos \left[(2 n-1) \cdot\left(\omega_{m}\left(t-\tau_{i}\right)-\frac{\omega_{m} \tau}{2}\right)\right]
\end{array}\right\}\right\}
\end{aligned}
$$


$2^{\text {nd }}$ harmonic

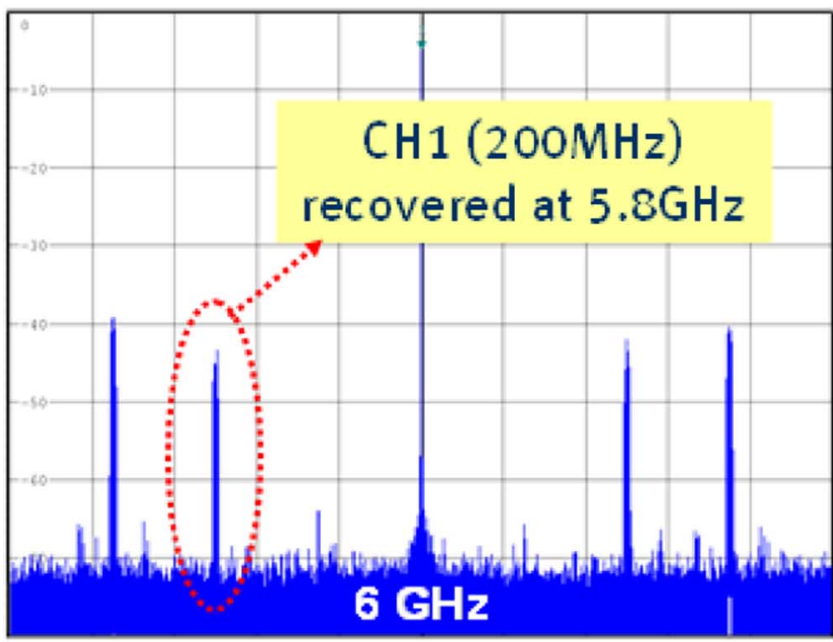

Center $\&$ aHz

fiber transmission.

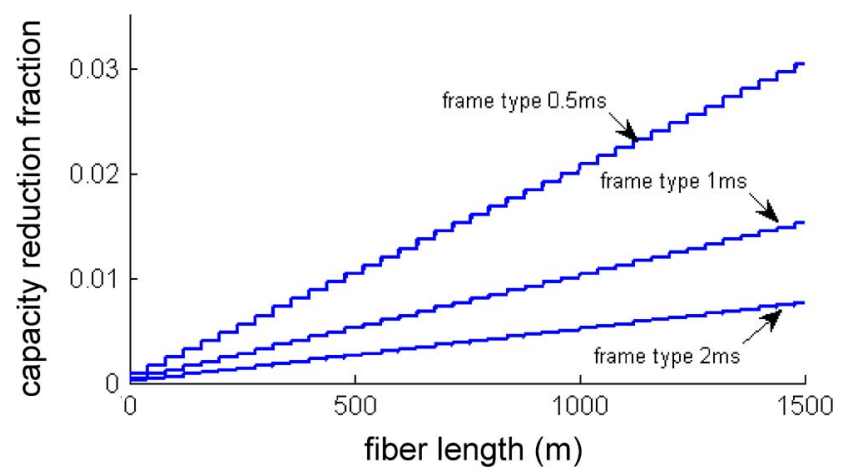

Fig. 13. IEEE 802.16 TDD frame capacity reduction for the accommodation of an optical fiber link between the CS and the AS (symbol rate is $10 \mathrm{MBaud}$ ).

Thus $i_{\text {out }}(t)$ contains even harmonic frequency components 2 $n \cdot \omega_{\mathrm{m}}$ with amplitude

$$
R_{d} P_{0} \cdot \cos \omega_{0} \tau \cdot J_{2 n}\left(2 \beta \cdot \sin \left(\omega_{m} \tau / 2\right)\right) \cdot\left|H_{\mathrm{IM}}\left(2 n \cdot \omega_{m}\right)\right|
$$

and odd harmonic frequency components $(2 n-1) \cdot \omega_{m}$ with amplitude

$$
\begin{aligned}
R_{d} P_{0} & \cdot \sin \omega_{0} \tau \\
\cdot & J_{2 n-1}\left(2 \beta \cdot \sin \left(\omega_{m} \tau / 2\right)\right) \cdot\left|H_{\mathrm{IM}}\left((2 n-1) \cdot \omega_{m}\right)\right| .
\end{aligned}
$$

Thus, due to the modal dispersion in the MMF, the amplitudes of the harmonics generated by the OFM process are scaled linearly with the amplitude of the MMF's intensity modulation transfer function. Note that this intensity modulation transfer function can have a significantly extended frequency characteristic in today's multimode graded-index fibers due to reduced differential mode delays by careful refractive index profile optimization (see also [13]), and by noncentral mode launching at the MMF's input [7].

When taking mode coupling into account, each guided mode may receive some signal contributions from other modes, which are delayed with respect to its own signal. Thus, within this mode an interference process occurs similar to the intentional interference process in the MZI in the OFM concept (see Fig. 7). Hence, in case of mode coupling the MMF itself also contributes to the FM-to-IM conversion of the OFM process. As long as the delay $\tau$ in the MZI considerably exceeds the differential mode delays in the MMF (which effectively are decreased by mode coupling, as this tends to average out mode delay differences), the MZI will be the dominant contributor to the OFM process. More details can be found in [14].

Next to being robust against modal dispersion in multimode fiber links, the OFM technique has also been shown to be robust against chromatic dispersion in SMF links (see [15]).

\section{ROMMF SYSTEM DESIGN USING OFM}

Exploiting the features of the OFM technique, a reliable RoF physical layer can be designed, comprising bidirectional RF transmission, increased cell capacity allocation, multistandard support, remote LO delivery, and an in-band control channel for dynamic radio link adaptation and remote antenna station controlling [16]. The proposed scheme can be easily integrated in wavelength-multiplexed network architectures, allowing a flexible convergence of wireless services with broadband access optical networks.

\section{A. Bidirectional RoF Transmission}

Next to delivering high capacity data on a clean microwave carrier in downstream direction to the AS, by employing the OFM technique also the upstream data path can be established. The downstream data, in, e.g., QAM format positioned on a subcarrier, is intensity-modulated on the frequency-swept signal in the CS. As shown in Fig. 9, the microwave carrier available at the AS can be down- (or up-) shifted by means of a mixer and a local low-frequency oscillator (LO). By mixing with this downshifted microwave, the upstream microwave received from the mobile terminal at the antenna is shifted to a low intermediate 


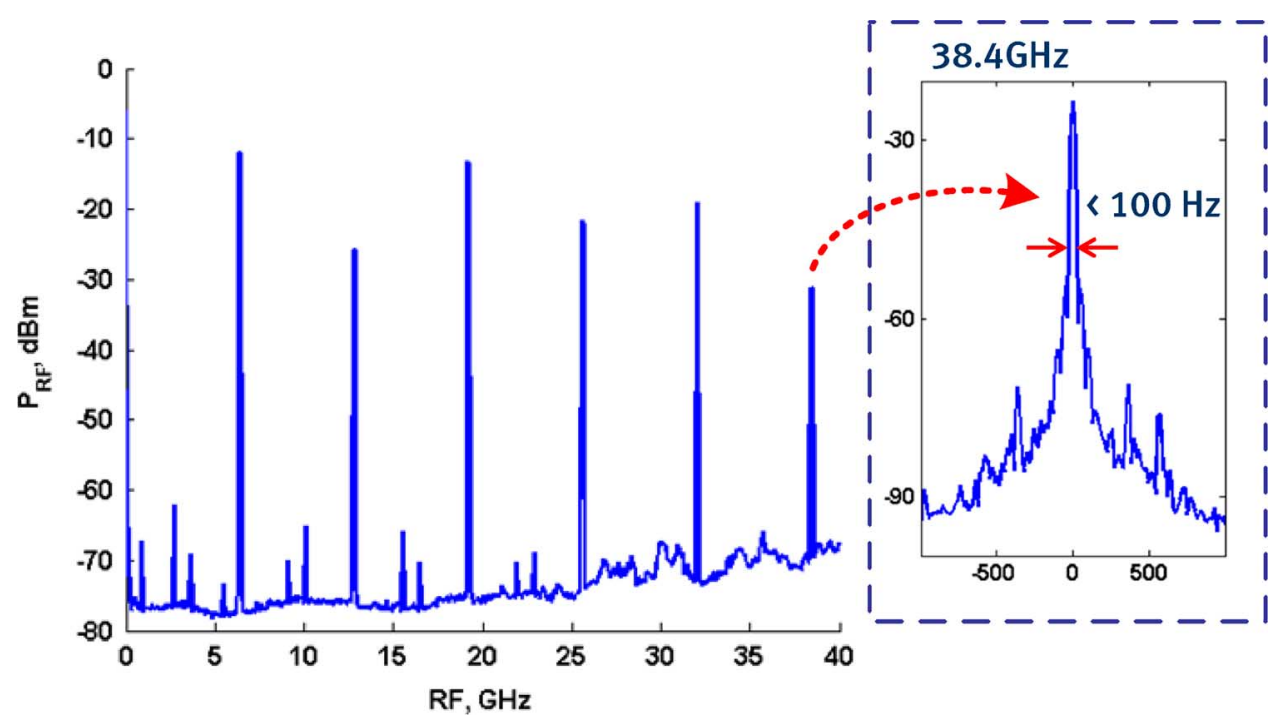

Fig. 14. Harmonics generation with $f_{\mathrm{sw}}=6.4 \mathrm{GHz}$. Inset: linewidth of the sixth harmonic (38.4 GHz) [20].

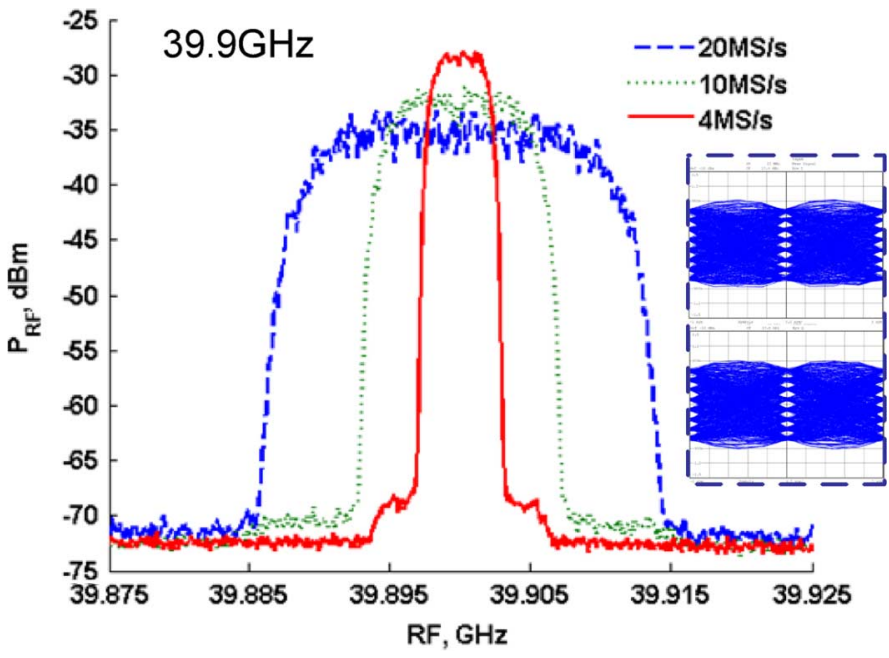

Fig. 15. 64-QAM signals upconverted to $39.9 \mathrm{GHz}$ by OFM with $f_{\mathrm{sw}}=6.4$ $\mathrm{GHz}$ and $f_{\mathrm{sc}}=1.5 \mathrm{GHz}$ (sixth harmonic). I-eye and Q-eye diagrams in the insets. $\mathrm{EVM}=4.88 \%$ for $120 \mathrm{Mb} / \mathrm{s}$. [20].

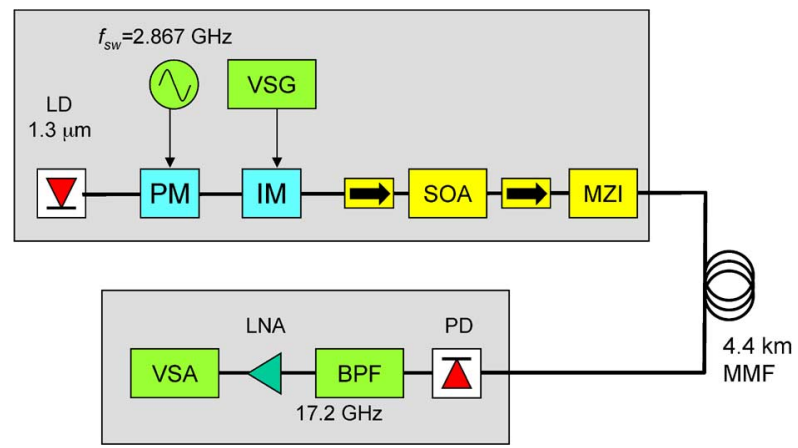

Fig. 16. QAM-64 $120 \mathrm{Mbit} / \mathrm{s}$ data delivery at $17.2 \mathrm{GHz}$ over a $4.4-\mathrm{km}$ silica MMF link (VSG: Vector Signal Generator, VSA: Vector Signal Analyser, PM: Phase Modulator, IM: Intensity Modulator).

frequency (IF) upstream carrier. Modulated on this one, the upstream data can be sent to the CS by a simple intensity modulation and direct detection scheme, using a low-cost low-fre- quency laser diode in the AS and photodetector in the CS. By remotely (from the CS, e.g., by an embedded control channel) tuning the local oscillator, the intermediate frequency of the upstream signal can be adapted individually per AS, which enables an FDMA upstream protocol for multiple AS-s.

The local oscillator signal at the AS can also be generated at the CS, and delivered by means of the OFM technique, using a pilot tone subcarrier separate from the data subcarrier, as shown in Fig. 10, [16]. At the photodetector output, the pilot subcarrier is upconverted together with the RF channels to $f_{\mathrm{LO}}=n$ • $f_{s w} \pm f_{\mathrm{sc}-\text { pilot }}$, and can be used as a local oscillator (LO) at the AS. Thus, the uplink RF channels arriving at the AS are mixed with the remotely delivered $\mathrm{LO}$ signals and downconverted to the uplink low IF $f_{\mathrm{UL}}=\left|f_{\mathrm{LO}}-f_{\mathrm{RF}}\right|$ for upstream transmission. For the sake of simplicity, also one of the generated harmonics $\left(n \bullet f_{\mathrm{sw}}\right)$ can be used as a LO at the AS, avoiding the necessity of the pilot subcarrier [17].

\section{B. Increased Cell Capacity Allocation and Multistandard Support}

In a RoF link employing OFM, the generated harmonics at the AS are spaced by the sweep frequency $f_{\mathrm{sw}}$, limiting in this way the maximum RF bandwidth available for transmission to $f_{\mathrm{sw}} / 2$. Thus, any radio signal at a low-frequency subcarrier $f_{\mathrm{sc}}<f_{\mathrm{sw}} / 2$ can be introduced by intensity modulation at the CS, transparently transmitted to the AS, and recovered upconverted in double-sideband modulation format along with the desired harmonic. When directly sweeping the laser wavelength, the maximum $f_{\mathrm{sw}}$ is limited by the FM response of the laser diode. When using an external phase modulator to impress the wavelength sweep, $f_{\mathrm{sw}}$ can be much larger. Provided that the maximum RF bandwidth $\left(f_{\mathrm{sw}} / 2\right)$ is not exceeded, different wireless signals can be transmitted simultaneously in a subcarrier multiplexing (SCM) scheme [18] (see Fig. 11). Thus, at the AS, the obtained upconverted signals can be selected at the same or at different harmonics bands. This makes it possible to increase the cell capacity of a wireless system at the AS, by selecting the signals in the same harmonic band. Also, a 


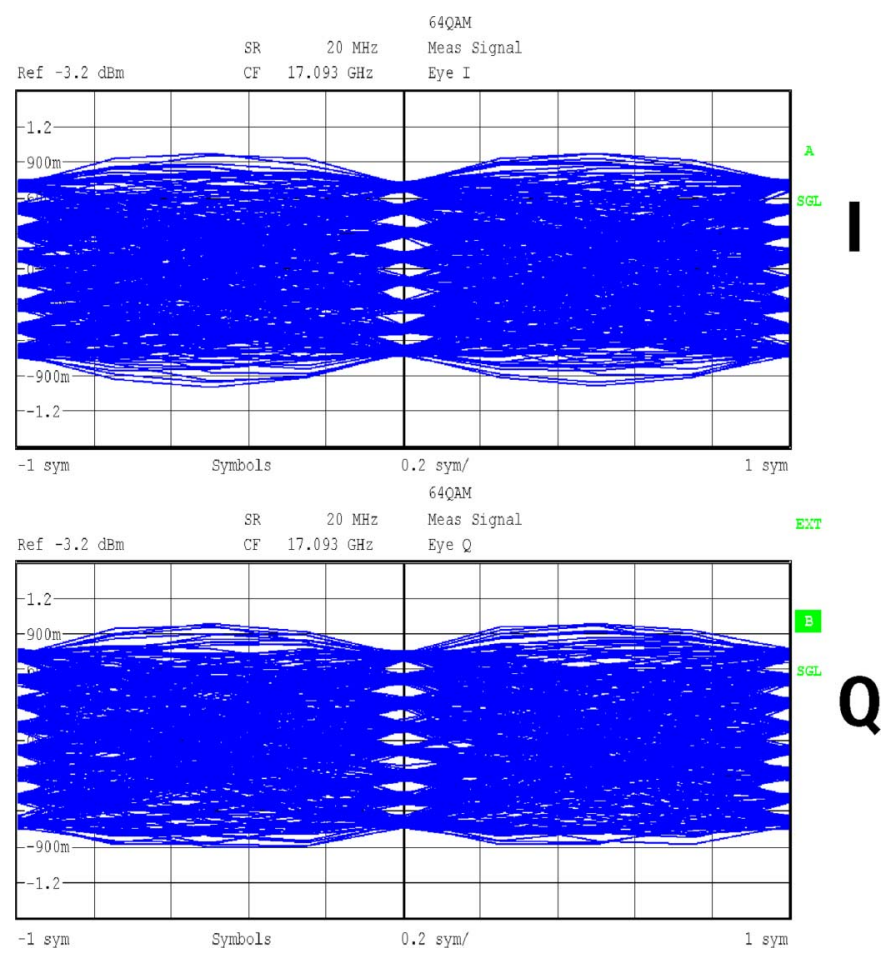

Fig. 17. Received eye patterns of the QAM-64 I- and Q-phase channels.

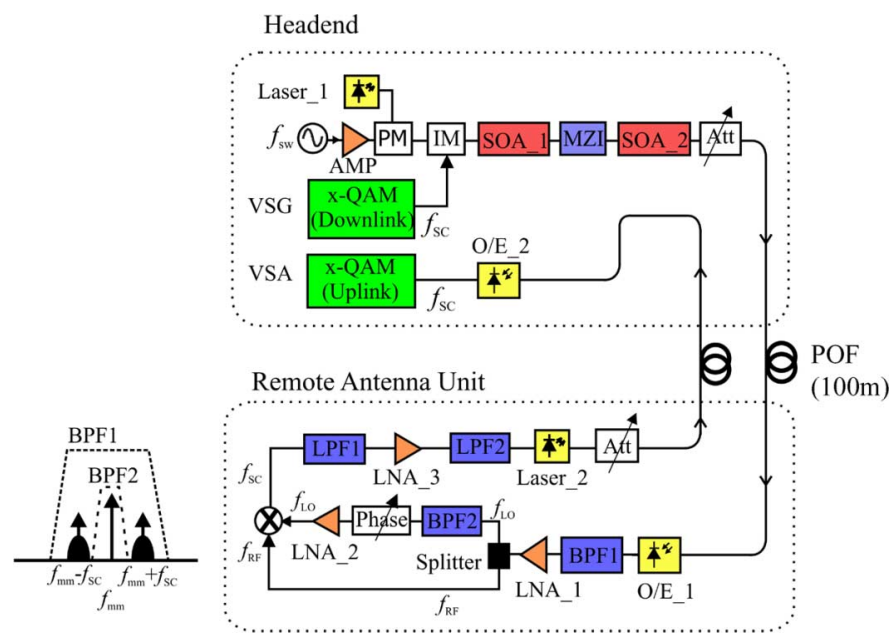

Fig. 18. Bidirectional radio-over-POF system using OFM.

proper election of the $f_{\mathrm{sw}}$ and $f_{\mathrm{sc}}-\mathrm{s}$ at the CS enables the simultaneous recovery of the wireless signals in different harmonic bands (see Fig. 12) [18]. In this way, multiple wireless standards can be simultaneously and transparently transmitted to the same AS, e.g., WiFi and WiMax, in a single OFM link, employing only one laser source and low-frequency electronics at the CS.

\section{Dynamic Radio Link Adaptation With OFM}

Dynamic radio link adaptation, i.e., the mechanisms by which modulation, coding and other signal and protocol parameters are dynamically adapted to the conditions of the radio link (e.g., path loss, interference, receiver sensitivity, available transmit power budget, etc.) in order to achieve optimum performance, is a key feature in wireless communication systems. Thus, a RoF link has to support this adaptability without incurring additional signal degradation along the optical path, being as independent as possible of the radio link adaptation procedures. Whereas link/MAC and baseband adaptation can be controlled from the $\mathrm{CS}$, the RF adaptation may occur either at the CS or at the AS. In this last case, adaptive remote AS configuration may be necessary. Hence, a tradeoff between $A S$-simplicity and minimum level of AS-intelligence needed arises. OFM enables a flexible mechanism for the dynamic radio link adaptation support [18]:

- Dynamic carrier frequency allocation can be easily performed from the CS by tuning low frequency subcarriers.

- Transmit power can be remotely controlled from the CS and adjusted at the AS, to alleviate optical dynamic range requirements in the RoF link; for this purpose, an in-band control channel has to be transmitted simultaneously with the wireless data channel from the CS to the AS.

In a more general approach, an in-band control channel in the same optical link may enable other mechanisms for remote antenna configuration and controlling during network optimization and dynamic resource allocation.

\section{Impact on Radio MAC Protocols}

When inserting a RoF link to support the current and emerging wireless standards, the multiple radio access mechanisms, as well as the radio duplexing schemes, become a key requirement for the design of the RoF distributed system. This is because the additional propagation delay introduced by the fiber link might outrun the timing boundaries of the medium access protocols and the round trip delay. Centrally scheduled MAC schemes, e.g., the one defined by IEEE 802.16 for fixed wireless access, allow flexibility for the insertion of an optical system between the CS and the AS, and for dynamically accommodating the additional propagation delay inserted by the fiber link by putting it into an increased guard time between the upstream and downstream timeslots. In the time division duplex (TDD) mode of operation, this additional propagation delay implies a frame capacity reduction, linearly increasing with the fiber length [19]. As shown in Fig. 13, for fiber spans shorter than $500 \mathrm{~m}$, this reduction is less than $1 \%$ for the three frame types defined by the standard. With longer fiber links, the 2-ms frame type is more efficient in terms of radio resource utilization, since the ratio of idle physical slots to the total number of physical slots is lower than in the shorter frame types.

\section{OFM SYSTEM EXPERIMENTS}

\section{A. Microwave Signal Generation and Modulation}

In order to assess the spectral characteristics of the OFM-generated microwaves, spectrum measurements have been made. As discussed in Section III-A, the impact of the laser phase noise is negligible when the laser linewidth is much smaller than a quarter of the Free Spectral Range $\Delta \omega_{\mathrm{FSR}}=2 \pi / \tau$ of the MZI. This condition is easily met, as the MZI's FSR is typically around $10 \mathrm{GHz}$, and, thus, the OFM process effectively suppresses the laser phase noise. Hence, very pure microwave signals can be generated. Linewidths below the measurement resolution $(<100 \mathrm{~Hz})$ have been obtained when generating a 
38.4-GHz carrier as the sixth harmonic of a $6.4-\mathrm{GHz}$ sweep signal, whereas the linewidth of the laser diode was more than $1 \mathrm{MHz}$; see [20]. Moreover, by using a high-order harmonic, OFM permits to use only a relatively low-frequency sweep generator in the CS, which can be of high quality while still at low cost.

The high purity of the generated microwave carriers allows the upconversion of radio signals with complex modulation formats (e.g., multilevel-QAM). For example, Fig. 15 shows the upconversion of 64-QAM signals from $1.5 \mathrm{GHz}$ to $39.9 \mathrm{GHz}$ (along with the sixth harmonic of $f_{\mathrm{sw}}=6.4 \mathrm{GHz}$ ), for which an EVM value of $4.88 \%$ was measured (this value lies well below the $5.6 \%$ specified for the maximum transmitter constellation error by the WLAN standard IEEE802.11a). As outlined before, the radio signal bandwidth available at each harmonic is $1 / 2 f_{\mathrm{sw}}=3.2 \mathrm{GHz}$.

\section{B. RoMMF Link}

The robustness of the OFM technique against modal dispersion was discussed in Section III-B. It has been demonstrated in a link of $4.4 \mathrm{~km}$ of silica multimode graded-index fiber with 50 $\mu \mathrm{m}$ core, using the laboratory setup shown in Fig. 16, [21].

The cleanliness of the $17.2-\mathrm{GHz}$ microwave carrier (measured FWHM spectral width is below measurement resolution of $100 \mathrm{~Hz}$ ) allowed to deploy a comprehensive signal modulation format. Using QAM-64 modulated with a symbol rate of $20 \mathrm{MBaud}$ on a subcarrier of $127 \mathrm{MHz}$ and subsequently transferred on the 17.2-GHz microwave carrier, a data transport rate of $120 \mathrm{Mbit} / \mathrm{s}$ was achieved over the 4.4-km MMF link. The received eye patterns of the In-phase and Quadrature-phase signals of the QAM-64 signal are shown in Fig. 17. The measured error vector magnitude (EVM) was $4.8 \%$, which is well below the 5.6\% value required for good QAM-64 detection. Even at a microwave carrier of $29.7 \mathrm{GHz}$, a rate of $60 \mathrm{Mbit} / \mathrm{s}$ using QAM-64 could be achieved over 4.4-km links of three different types of silica $50-\mu \mathrm{m}$ core graded index MMF link [13]. The limitation to the microwave frequency was set by the bandwidth of the photodiode $(25 \mathrm{GHz}$ at $-3 \mathrm{~dB})$, rather than by the MMF. Using graded-index POF with $120-\mu \mathrm{m}$ core diameter, a similar system setup has been reported in [22], where a $16-\mathrm{GHz}$ microwave carrier was delivered over a 330-m fiber link.

\section{Bidirectional RoMMF System}

Based on the schemes showed in Figs. 9 and 10, RoMMF bidirectional transmission has been demonstrated over graded-index silica MMF [17] and over 50- $\mu \mathrm{m}$ core graded-index POF [23]. Fig. 18 shows the experimental setup schematic used in [23] for bidirectional transmission over POF. The 1316-nm DFB laser diode at the CS emits 10-mW continuous wave light, of which the optical frequency is swept sinusoidally by a phase modulator (PM) driven by a $2.867-\mathrm{GHz}$ sweep signal. The signal was subsequently intensity-modulated by a 100-Mbit/s QAM-16 signal from the vector signal generator (VSG), modulated on a subcarrier with frequency $f_{\mathrm{sc}}=300 \mathrm{MHz}$. The MZI performing the OFM process is put between two semiconductor optical amplifiers for power boosting. After $100 \mathrm{~m}$ of $50-\mu \mathrm{m}$ core graded-index POF, the signal was coupled at the remote antenna unit site into a 50- $\mu \mathrm{m}$

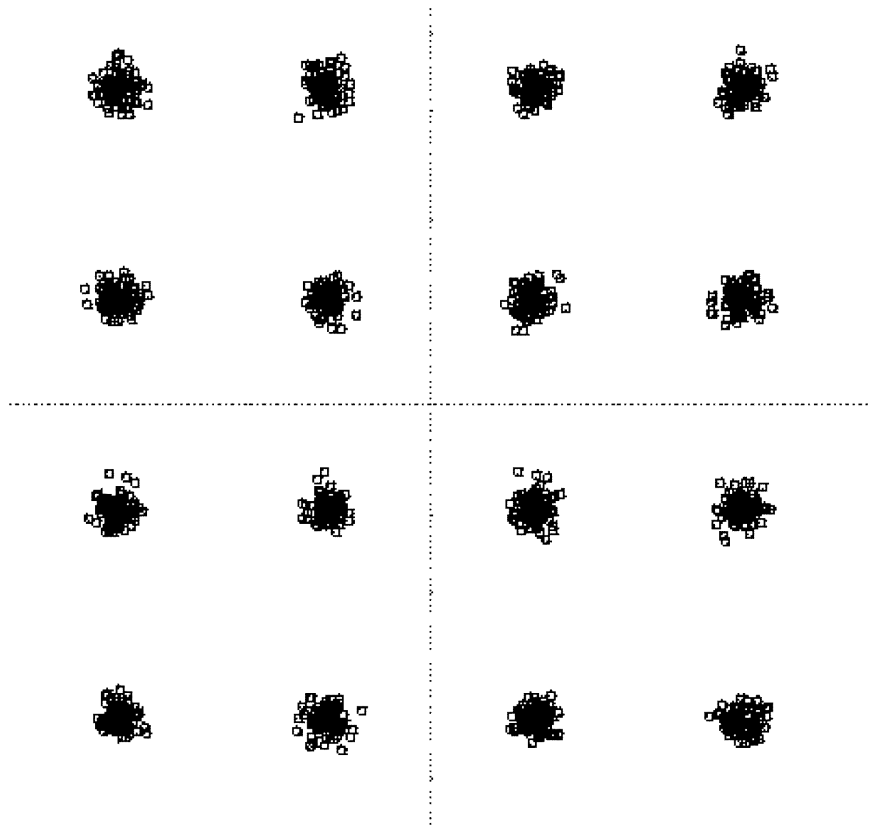

Fig. 19. Constellation diagram of 100-Mbit/s QAM-16 received uplink signal.

core MMF pigtailed photodiode having a bandwidth of 25 GHz. Thereafter, an electrical bandpass filter BPF1 filters the desired 17.2- $\mathrm{GHz}$ microwave carrier plus its QAM-modulated subcarriers. By means of a second narrowband electrical bandpass filter BPF2, the 17.2-microwave carrier could be separated and used for downmixing the downstream signal. The resulting QAM-modulated IF signal at frequency $f_{s c}$ was used as low-frequency radio signal for the RoMMF uplink transmission. This uplink transmission was performed over a separate $100-\mathrm{m} 50-\mu \mathrm{m}$ GI-POF link using intensity modulation of a low bandwidth laser diode and direct detection.

Thus, including the downmixing and upstream transmission, the 16-QAM signal has made a round trip through 200-m GI-POF. The received constellation diagram observed with the VSA (see Fig. 19) showed an EVM of only $4.8 \%$, which denotes the high performance provided by OFM in downlink even over POF and the high quality of the generated microwave carriers to be used as a remotely delivered LO to the AS.

\section{RECONFIGURABLE RoF N-HOUSE NETWORKS}

Similarly as in the access network [24], optical routing in the in-building network may improve the flexibility and the efficiency with which the network resources can be used. By creating picocells for broadband wireless communication at frequencies needing line-of-sight (e.g., $60 \mathrm{GHz}$ ), communication is confined to a single room. However, as illustrated in Fig. 20, the transparent transport of those microwave radio signals by means of RoMMF techniques can enable communication among two or more rooms [25]. Each room can have one or more antennas, which by means of an optical fiber network and an optically transparent switch in the Home Communication Controller (HCC) site can be interconnected. In this way, several virtual private wireless LAN-s can be set up which do not interfere with each other. The HCC directs the RoF signals to the appropriate rooms, and by changing the switch settings the 


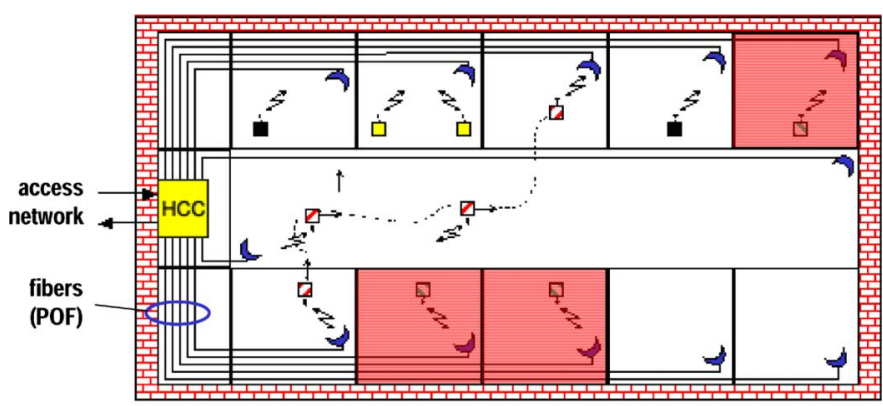

HCC: Home Communication Controller

Fig. 20. Flexible interroom wireless communication by optically switched RoF links.

configuration of the private WLANs can be adjusted readily. The optical transparency of this concept also allows multiple wireless standards (WiFi, WiMAX, 60-GHz LANs, ...) for in-building communication to coexist, and to be reconfigured easily in response to user demands.

This in-building network can also be based on a multiwavelength MMF bus or ring topology, with MMF-based wavelength add/drop nodes connecting the AS-s. As shown in Fig. 21, [27], at the CS, several RoMMF channels are wavelength multiplexed $\left(\lambda_{1}\right.$ to $\left.\lambda_{N}\right)$ and launched into the multimode fiber ring. The wavelength-selecting multimode add/drop node at the AS is built with a narrowband multimode FBG, as indicated in Fig. 22. Here, the WDM channels arriving at the AS pass through a multimode optical circulator to the multimode FBG, where the channel-to-drop $\lambda_{\mathrm{DLx}}$ (downlink for $A S_{\mathrm{x}}$ ) is reflected back to the circulator and dropped to the optoelectronic receiver in the $\mathrm{AS}_{\mathrm{x}}$. The multimode FBG lets pass the rest of the WDM RoMMF channels to the next AS. Since the $\lambda_{\mathrm{DLx}}-\mathrm{RoMMF}$ channel is removed from the set of WDM channels in the ring, a new uplink channel can be added to the ring at the same wavelength $\lambda_{\mathrm{ULx}}=\lambda_{\mathrm{DLx}}$. The uplink channel $\lambda_{\mathrm{DLx}}$ from $\mathrm{AS}_{\mathrm{x}}$ is added through the second optical circulator and reflected back in the multimode FBG to the ring in the same direction as the rest of the WDM RoMMF channels to be sent back to the CS.

The experimental arrangement in Fig. 22 was set up in order to study the feasibility and system performance of this scheme. At the CS, three laser sources formed the continuous wave input of the OFM signal processing for downlink transmission. These three optical channels were simultaneously frequency modulated by a sweep frequency of $6 \mathrm{GHz}$ using an optical phase modulator, and then intensity modulated by a $120-\mathrm{Mb} / \mathrm{s}$ 64-QAM radio data signal at low-frequency subcarrier of $300 \mathrm{MHz}$. The resulting optical signal was passed through a delay interferometer with delay $\tau=25 \mathrm{ps}$ to perform the FM-IM conversion, launched into $2.5 \mathrm{~km}$ of single-mode dispersion-shifted fiber to decorrelate the data signals in the optical channels, and preamplified with a semiconductor optical amplifier before entering the MMF ring. At the AS, a multimode add/drop multiplexer was built with commercially available $50-\mu \mathrm{m}$ core diameter multimode fiber components, consisting of two optical circulators and a multimode FBG of $97 \%$ reflectivity (15-dB out-of-band rejection) and $100-\mathrm{GHz}$ nominal bandwidth. For the uplink, a laser source was intensity

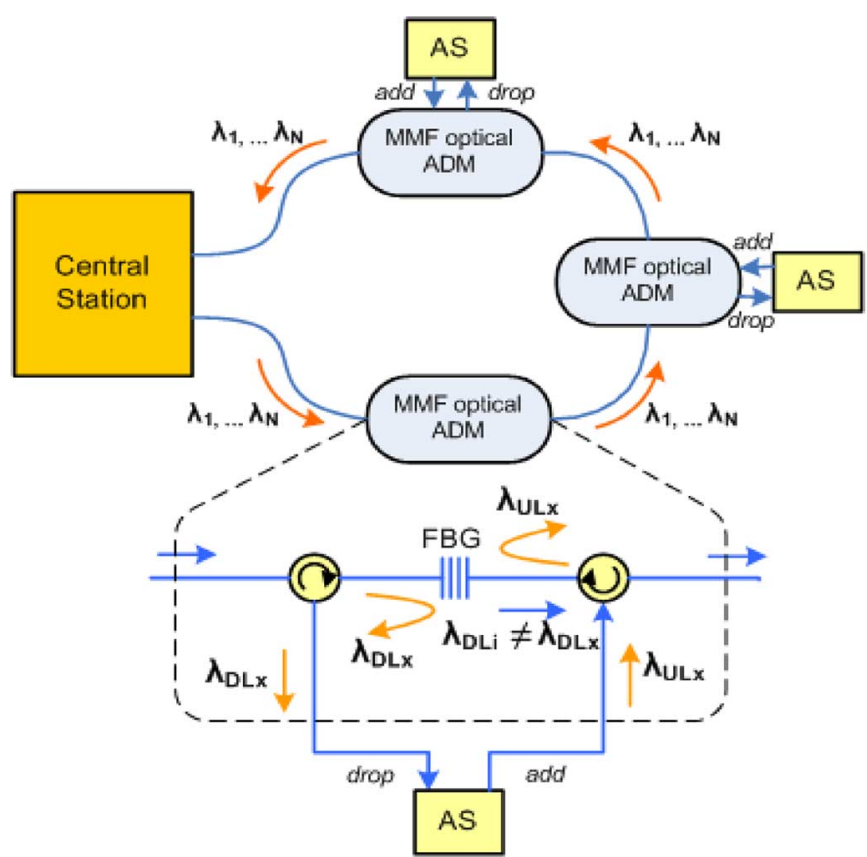

Fig. 21. All-fiber wavelength-division-multiplexing ring architecture for RoMMF distribution of broadband wireless services (MMF: multimode fiber, AS: antenna station, ADM: add-drop multiplexer, FBG: fiber Bragg grating).[27]

modulated by a $120-\mathrm{Mb} / \mathrm{s} 64-\mathrm{QAM}$ radio data signal at low-frequency $f_{\mathrm{IF}}=300 \mathrm{MHz}$. The resulting uplink channel was added to the MMF ring through the second optical circulator (OC2 in Fig. 22).

Fig. 23 shows the optical reflection and transmission spectra at the dropped and through ports (before OC2), respectively, of the add/drop node that selects the 1314.8-nm wavelength channel, when the add/drop node was injected with a white light source. Downlink and uplink 64-QAM signals were recovered with an optoelectronic receiver consisting of a variable optical attenuator and a MMF-pigtailed 25-GHz-bandwidth photodetector, and then analyzed with a vector signal analyzer: in downlink, the signal recovered at $f_{\mathrm{RF}}=23.7 \mathrm{GHz}$ (lower side band of the fourth harmonic, $24 \mathrm{GHz}$ ) was selected for the analysis; in uplink, the signal at $\mathrm{f}_{\mathrm{IF}}=300 \mathrm{MHz}$ was recovered by direct detection. This frequency selection accounts for the frequency bands allocated for short range devices (SRDs) and fixed wireless access (FWA) by the Dutch National Frequency Plan. The downlink and uplink transmission was performed over $4.4 \mathrm{~km}$ of $50-\mu \mathrm{m}$ core diameter silica graded index MMF with a bandwidth-distance product of $1.47 \mathrm{GHz} \cdot \mathrm{km}$ and typical $0.49-\mathrm{dB} / \mathrm{km}$ attenuation loss at $1310 \mathrm{~nm}$.

After downlink data transmission, the dropped $120-\mathrm{Mb} / \mathrm{s}$ 64-QAM radio signals at $23.7 \mathrm{GHz}$ were recovered with EVM values below $4.5 \%$. For the channel spacing applied here, a SNR penalty of up to $0.6 \mathrm{~dB}$ due to crosstalk was observed in downlink transmission. In uplink, the $120-\mathrm{Mb} / \mathrm{s} 64-\mathrm{QAM}$ radio signal at $300 \mathrm{MHz}$ was deteriorated $4.1 \mathrm{~dB}$ due to crosstalk from the corresponding dropped downlink channel, which is determined by the transmission and reflection characteristics of the multimode FBG. An additional 1.2-dB SNR penalty was observed after MMF transmission, which corresponds to the 

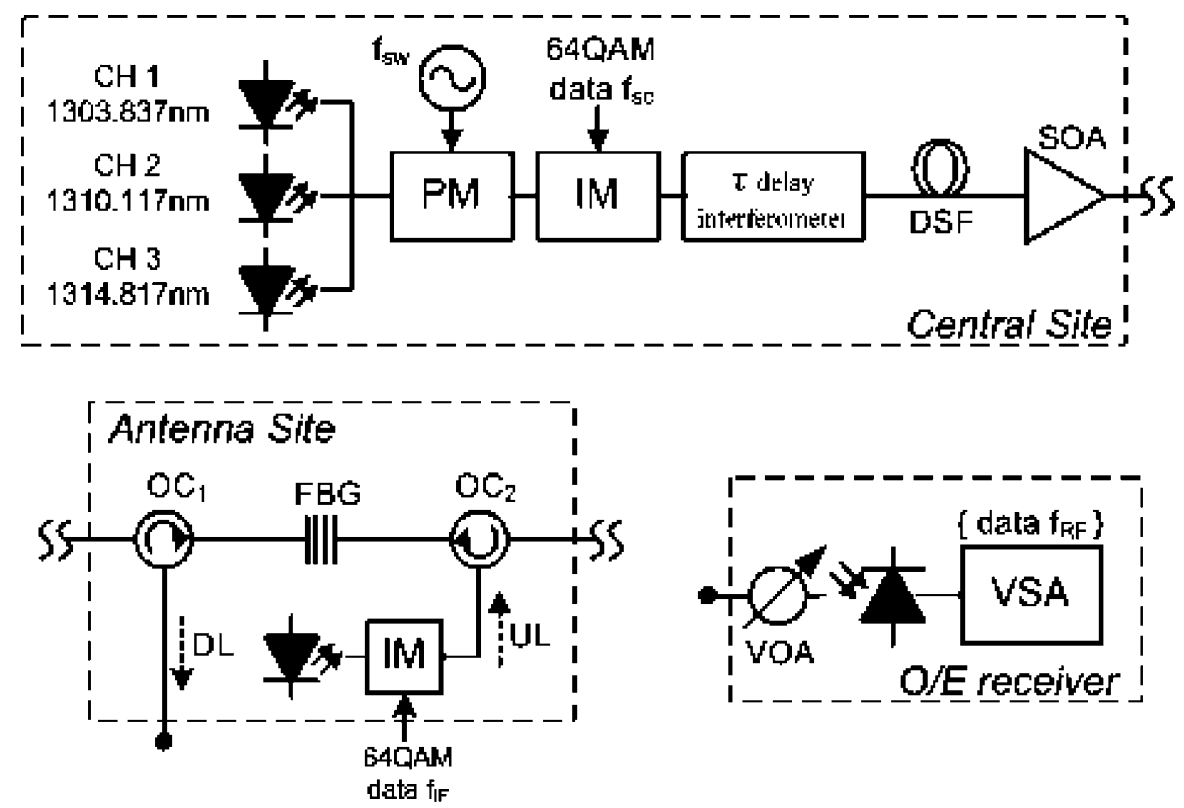

Fig. 22. Experimental setup schematics of the central station, the multimode add/drop node at the antenna site and the optoelectronic (O/E) receiver (PM: phase modulator; IM: intensity modulator; DSF: dispersion shifted fiber; SOA: semiconductor optical amplifier; OC: optical circulator; FBG: fiber Bragg grating; DL: downlink; UL: uplink; VOA: variable optical attenuator; VSA: vector signal analyzer).[27].
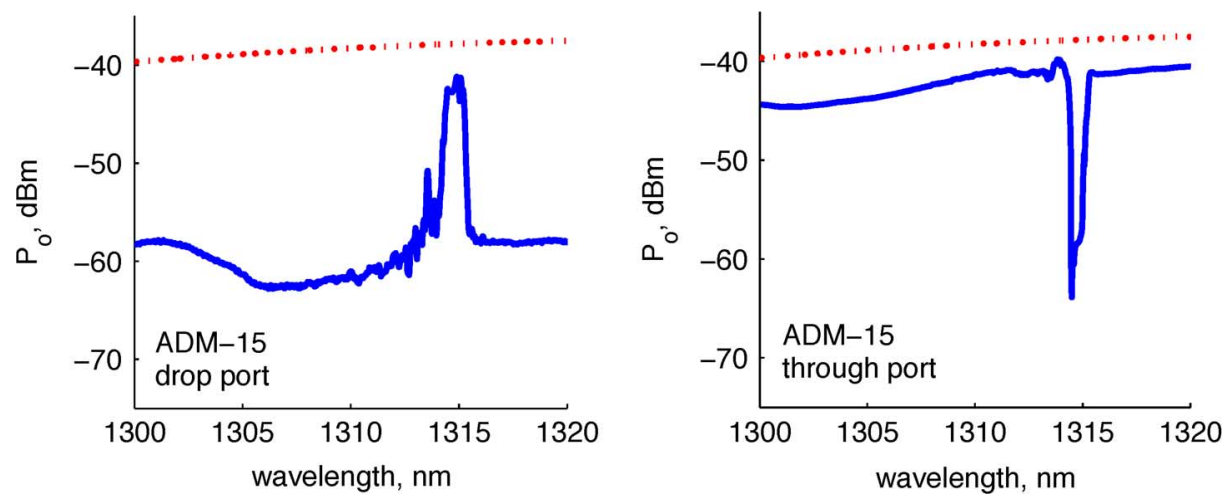

Fig. 23. ADM reflection and transmission spectra (dropped and through ports, respectively) of the add/drop node that selects a wavelength channel at 1314.8 nm, measured with a white light source; the dotted line indicates the input white light source spectrum.[27].

expected SNR penalty due to the MMF bandwidth in the direct transmission of a $300-\mathrm{MHz}$ carrier over $4.4 \mathrm{~km}(1.47-\mathrm{GHz} \mathrm{km}$ bandwidth-distance product).

Our experimental results show that the system performance is not jeopardized by modal dispersion, and that the robustness of the approach against modal dispersion impairments in MMF transmission is also maintained through the multimode add/drop node. This demonstrates the feasibility of a robust RoMMF in-building network. Still, the transmission and reflection characteristics of the FBG in the add/drop nodes are the major limitation of system performance. Therefore, further development of high-quality narrowband multimode FBG would improve the performance and reliability of the proposed multimode WDM network for RoMMF distribution.

\section{CONCLUSION}

For in-building service-integrated networks, multimode fiber (and, in particular, POF) constitutes a versatile backbone infrastructure which can carry both wirebound and wireless broadband services. Dispersion-robust RoF techniques are needed for the distribution of microwave signals in multimode fiber links. Both theoretically and experimentally, the optical frequency multiplying technique has shown its capabilities to generate microwave carriers with high spectral purity, and to overcome the multimode fiber's modal dispersion. The feasibility has been demonstrated by transporting $120 \mathrm{Mbit} / \mathrm{s}$ QAM-64 signals up to $30 \mathrm{GHz}$ over $4.4 \mathrm{~km}$ of graded-index $50 \mu \mathrm{m}$ core silica MMF, and 100-Mbit/s QAM-16 at carrier frequencies beyond $17 \mathrm{GHz}$ over $100 \mathrm{~m}$ of graded-index 120 $\mu \mathrm{m}$ core POF. RoMMF link bidirectionality, simultaneous multiple radio standards support and point-to-multipoint distribution schemes have been experimentally demonstrated as well. By means of signal-transparent optical cross-connect switching of the RoF signals, reconfigurable multistandard wireless multipicocell LANs can be created, which creates new network opportunities such as flexible interroom wireless communication. 


\section{ACKNOWLEDGMENT}

The authors would like to thank their co-workers J. Zeng, J.-J. Vegas Olmos, H. Yang, Y. Zheng, A. Ng'oma, and G.-J. Rijckenberg for valuable contributions.

\section{REFERENCES}

[1] A. M. J. Koonen, A. Ng'oma, G.-J. Rijckenberg, M. G. Larrode, P. J. Urban, H. de Waardt, J. Yang, H. Yang, and H. P. A. van den Boom, "How deep should fibre go into the access network?," in Proc. ECOC 2007, Berlin, Germany, Sep. 16-20, 2007, paper Mo1.1.4.

[2] A. Flatman, "In-premises optical fiber installed base analysis to 2007," presented at the IEEE 802.310 GBE over FDDI Grade Fiber Study Group, Orlando, FL, Mar. 2004, presented at the, unpublished.

[3] D. Wake, S. Dupont, C. Lethien, J. P. Vilcot, and D. Decoster, "Radio frequency transmission of 32-QAM signals over multimode fiber for distributed antenna system applications," Inst. Elect. Eng. Electron. Lett., vol. 37, no. 17, pp. 1087-1089, Aug. 2001.

[4] C. Carlsson, A. Larsson, and A. Alping, "RF transmission over multimode fibers using VCSELs - Comparing standard and high-bandwidth multimode fibers," J. Lightw. Technol., vol. 22, no. 7, pp. 1694-1700, Jul. 2004.

[5] A. Das, A. Nkansah, N. J. Gomes, I. J. Garcia, J. C. Batchelor, and D. Wake, "Design of low-cost multimode fiber-fed indoor wireless networks," IEEE Trans. Microw. Theory Tech., vol. 54, no. 8, pp. 3426-3432, Aug. 2006

[6] P. Hartmann, X. Qian, A. Wonfor, R. V. Penty, and I. H. White, "1-20 GHz directly modulated radio over MMF link," presented at the Proc. Int. Topical Meet. Microw. Photon. (MWP'05), Oct. 2005.

[7] N. Gomes, A. Nkansah, and D. Wake, "Radio over MMF techniques, Part I: RF to microwave frequency systems," J. Lightw. Technol., vol. 26, no. 15, pp. 2388-2395, Aug. 1, 2008.

[8] R. A. Griffin, P. M. Lane, and J. J. O'Reilly, "Radio-over-fiber distribution using an optical millimeter-wave/DWDM overlay," presented at the Proc. OFC'99, San Diego, CA, Feb. 22-25, 1999, paper WD6.

[9] A. M. J. Koonen and A. Ngoma, "Integrated broadband optical fibre/ wireless LAN access networks," in Broadband Optical Access Networks and Fiber-to-the-Home: System Technologies and Development Strategies. New York: Wiley, 2006.

[10] T. Ismail, C.-P. Liu, J. Mitchell, A. Seeds, X. Qian, A. Wonfor, R Penty, and I. White, "Bidirectional transmission of broadband wireless signals using a millimeter-wave over fibre CWDM ring architecture," presented at the Proc. NEFERTITI Symp., Brussels, Belgium, Jan. $17-19,2005$

[11] A. M. J. Koonen, A. Ng'oma, H. P. A. van den Boom, I. Tafur-Monroy, P. F. M. Smulders, and G.-D. Khoe, "Carrying microwave signals in a GIPOF-based wireless LAN," in Proc. POF 2001, Amsterdam, Sept. 27-30, 2001, pp. 217-223.

[12] T. Koonen, A. Ng'oma, P. Smulders, H. van den Boom, I. T. Monroy, P. van Bennekom, and G.-D. Khoe, "In-house networks using polymer optical fibre for broadband wireless applications," in Proc. ISSLS 2002 (XIVth Int. Symp. Services and Local Access), Seoul, Korea, Apr. 14-18, 2002, pp. 285-294, paper 9-3.

[13] M. G. Larrode, A. M. J. Koonen, and J. J. V. Olmos, "Overcoming modal bandwidth limitation in radio-over-multimode fiber links," IEEE Photon. Technol. Lett., vol. 18, no. 22, pp. 2428-2430, Nov. 2006.

[14] M. G. Larrode and A. M. J. Koonen, "Theoretical and experimental demonstration of OFM robustness against modal dispersion impairments in radio over multimode fiber links," J. Lightw. Technol., accepted for publication.

[15] A. M. J. Koonen, M. G. Larrode, A. Ng'oma, K. Wang, H. Yang, Y. Zheng, and E. Tangdiongga, "Perspectives of radio over fiber technologies," presented at the Proc. OFC 2008, San Diego, CA, Feb. 24-28, 2008, paper OThP3

[16] M. G. Larrode and A. M. J. Koonen, "Physical layer design for RoFbased wireless access networks," presented at the Proc. ECOC 2006, Cannes, France, Sep. 24-28, 2006, paper We3.P.167.

[17] M. G. Larrode, A. M. J. Koonen, J. J. V. Olmos, and A. NgOma, "Bidirectional radio-over-fiber link employing optical frequency multiplication," IEEE Photon. Technol. Lett., vol. 18, no. 1, pp. 241-243, Jan. 2006.

[18] M. G. Larrode, A. M. J. Koonen, and J. J. V. Olmos, "Fiber-based broadband wireless access employing optical frequency multiplication," IEEE J. Sel. Topics Quantum Electron., vol. 12, no. 4, pp. 875-881, Jul. 2006.

[19] M. G. Larrode, A. M. J. Koonen, J. J. V. Olmos, G. J. Rijckenberg, B. L. Dang, and I. G. M. M. Niemegeers, "Transparent transport of wireless communication signals in radio-over-fibre systems," in Proc. 10th Europ. Conf. Netw. Opt. Commun., NOC2005, London, U.K., Jul. 2005.
[20] M. G. Larrode, A. M. J. Koonen, J. J. V. Olmos, E. J. M. Verdurmen, and J.P. Turkiewicz, "Dispersion tolerant radio-over-fibre transmission of 16 and 64 QAM radio signals at $40 \mathrm{GHz}$," Inst. Elect. Eng. Electron. Lett., vol. 42, no. 15, pp. 872-874, Jul. 2006.

[21] A. Ng'oma, A. M. J. Koonen, I. Tafur-Monroy, H. P. A. v. d. Boom, and G. D. Khoe, "Using optical frequency multiplication to deliver a $17 \mathrm{GHz} 64$ QAM modulated signal to a simplified radio access unit fed by multimode fiber," in Proc. OFC 2005, Anaheim, CA, Mar. 6-11, 2005, paper OWB2.

[22] A. Ng'oma, I. Tafur-Monroy, A. M. J. Koonen, H. P. A. van den Boom, P. F. M. Smulders, and G.-D. Khoe, "High-frequency carrier delivery to graded-index polymer optical fibre fed next generation wireless LAN radio access points," presented at the Proc. ECOC 2003, Rimini, Sep. 21-25, 2003, paper We3.4.2.

[23] A. Ng'oma, J. Zeng, H. P. A. van den Boom, Y. Watanabe, and A. M. J. Koonen, "Bi-directional polymer optical fiber-based system for distributing 100 Mbps WiMAX signals exceeding $17 \mathrm{GHz}$," in Proc. Asian-Pacific Microw Photon Conf. (APMWP) 2007, Jeju Island, South Korea, Apr. 25-27, 2007.

[24] T. Koonen, "Fiber to the home/fiber to the premises: What, where, and when?," Proc. IEEE, vol. 94, no. 5, pp. 911-934, May 2006.

[25] A. M. J. Koonen, M. G. Larrode, J. Yang, A. Ng'oma, G.-J. Rijckenberg, P. Urban, H. Yang, H. P. A. van den Boom, and H. De Waardt, "Recent research results on flexible optical access and in-building networks," in Proc. NOC 2007, Stockholm, Sweden, Jun. 19-21, 2007, pp. $153-160$

[26] M. G. Larrode, A. M. J. Koonen, J. J. V. Olmos, and E. J. M. Verdurmen, "OFM performance in a basic MMF dropping node for radio-over-MMF ring/bus distribution antenna system," presented at the Proc. Asian-Pacific Microw. Photon. Conf. (APMWP) 2007, Jeju Island, South Korea, Apr. 25-27, 2007, session J3.

[27] M. G. Larrode and A. M. J. Koonen, "All-fiber full-duplex multimode wavelength-division-multiplexing network for radio-over-multimodefiber distribution of broadband wireless services," IEEE Trans. Microw. Theory Tech., vol. 56, no. 1, pp. 248-255, Jan. 2008.

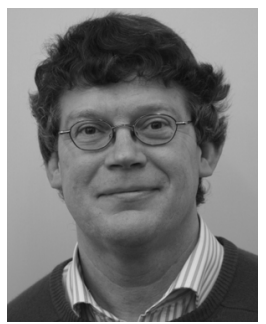

A. M. J. Koonen (M'00-SM'01-F'07) received the M.Sc. degree (cum laude) in electrical engineering from Eindhoven University of Technology, Eindhoven, The Netherlands, in 1979.

He was with Bell Laboratories, Lucent Technologies, as a Technical Manager of Applied Research for more than 20 years. He was a part-time Professor with Twente University, Enschede, The Netherlands, from 1991 to 2000. Since 2001, he has been a Full Professor with the Electro-optical Communication Systems Group, COBRA Institute, Eindhoven University of Technology, where he has been the Chairman of this group since 2004. His current research interests include broadband fiber access and in-building networks, radio-over-fiber networks, and optical packet-switched networks. He has initiated and led several European and national R\&D projects in this area on dynamically reconfigurable hybrid fiber access networks, fiber-wireless, packet-switched access, and short-range multimode (polymer) optical fiber networks, and label-controlled optical packet routed networks. Currently, he is involved in a number of access/in-home projects in the Dutch Freeband program, the Dutch IOP Generieke Communicatie program, and the EC FP6 IST and FP7 ICT programs.

Prof. Koonen is a Bell Laboratories Fellow since 1998 and a member of the LEOS Board of Governors since 2007.

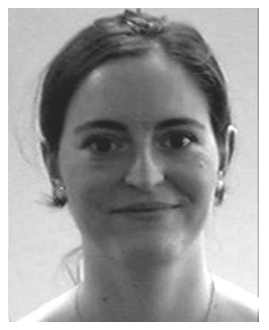

M. García Larrodé (S'04) was born in Zaragoza, Spain, in 1977. She received the M.Sc. degree in telecommunications engineering from the Centro Politécnico Superior, University of Zaragoza, in 2001

She is currently working toward the Ph.D. degree in broadband wireless access networks employing radio-over-fiber techniques with the COBRA Research Institute, Eindhoven University of Technology, Eindhoven, The Netherlands. From 2000 to 2004, she was a Systems Engineer with Siemens AG, Munich, Germany, where she was involved with mobile radio access networks with a focus on radio resource management, signaling, and performance evaluation of GSM/GPRS/EDGE, and UMTS networks. 